\section{Mental health in cystic fibrosis: turning the tide}

\author{
Trudy Havermans, ${ }^{1}$ Doris Staab ${ }^{2}$
}

In this issue the keenly awaited Mental Health Guidelines in Cystic Fibrosis (CF) are published. ${ }^{1}$ These guidelines are the result of the work of an international group of experts formed by the Cystic Fibrosis Foundation (CFF) and the European Cystic Fibrosis Society (ECFS). Fifteen years ago, the UK CF Trust Standards of Care described the important role played by a clinical psychologist as part of the CF multidisciplinary team. ${ }^{2}$ This role was reinforced in the ECFS 2005 Standards of Care document. ${ }^{3}$ The updated ECFS Best Practice Guidelines, published last year, made recommendations for surveillance and interventions to support patients and families with mental health problems. ${ }^{4}$ The CF community can be proud of their constant interest in and awareness of the psychological wellbeing of the patients they care for. In research, for more than 20 years a wide spectrum of topics have been studied, including quality of life, burden of treatment, treatment adherence, coping, life satisfaction, reproductive and sexual health, parenting, transition, end-of-life care etc. A recent (personal communication) survey by the Psychosocial Special Interest Group (working group of the ECFS) showed that 71 psychologist have dedicated hours to care for patients with CF (ranging from $10 \%$ to $100 \%$ ) in 14 European countries. However, from a recent survey ${ }^{5}$ we also know that mental health care delivery in $\mathrm{CF}$ is inadequate and highly variable across care centres and countries. This survey was distributed by the CFF and ECFS to approximately $4000 \mathrm{CF}$ health professionals. A total of 1454 professionals replied $(44.8 \%$ USA, $48.9 \%$ Europe), including mostly physicians, physiotherapists and nurses. The result showed that more than $20 \%$ had no one on their team whose primary role was mental health. The majority did not have a colleague trained to manage mental health issues or any experience with mental health screenings. Approximately

\footnotetext{
${ }^{1}$ Cystic Fibrosis Centre, University Hospital Leuven, Leuven, Belgium; ${ }^{2}$ Paediatric Pulmonologie and Allergology, Charité Berlin, Berlin, Germany
}

Correspondence to Trudy Havermans, Cystic Fibrosis Centre, University Hospital Leuven, Leuven 3000, Belgium, trudy.havermans@uzleuven.be
$30 \%$ felt unable or unsure if they could refer to mental health clinicians in their hospital and half did not have an up-to-date list of mental health resources and referrals. The respondents felt they needed funds to support a mental health professional; access to mental health resources and referrals; training in mental health screening; access to electronic tools for administration; and access to a mental health 'hotline' for guidance.

Approximately 8 years ago the CF community, initiated by Alexandra Quittner in the USA and Allister Duff in Europe, joined forces to specifically study anxiety and depression in CF (the TIDES study). ${ }^{6}$ The results showed that symptoms of depression and especially anxiety were elevated in patients with CF and in parents of children with CF. These results motivated the CFF and the ECFS to form the International Working Group on Mental Health to investigate the need and feasibility to write guidelines on mental health in CF.

For a guideline to be useful and effective, it should empower CF teams to give more effective care to patients and families with mental health problems and facilitate quality improvement. Guidelines may also allow CF teams to leverage funding for mental health professionals in health care systems where this has traditionally been overlooked.

The presented guidelines are set up by a wide panel of experts, professionals and patients/parents, and will facilitate each of these objectives. They are clear and readily applicable to the day-to-day reality of the clinic. To develop the guidelines, the working group focused on screening, psychological interventions, pharmacological treatments and implementation and future research. For each topic, specific questions were identified using a robust format described by Robinson et $\quad a l:^{7}$ Population, Intervention, Comparison, Outcome (PICO). The questions derived from this method were used for an extensive literature search. This resulted in a list of recommendations and $80 \%$ agreement was required for a recommendation to be included in the guidelines. In short, recommendations are made with regard to four themes: prevention, screening, clinical assessment and psychological and/or pharmacological interventions. Recommendations on prevention focus on the task of CF teams to provide sufficient information and education to help patients/parents to develop coping skills and disease management. Annual screening for depression and anxiety is recommended for patients (12 years and older) and parents (of children under the age of 17 years). When screening results indicate elevated scores for depression and/or anxiety, further clinical assessment is recommended followed, when indicated, by interventions from a psychological and a pharmacological perspective. The authors provide a flexible stepped-care model for the screening process and ample documentation is included to assist CF teams in implementing the guidelines and in quality improvement. Undoubtedly, the implementation will demand time and expertise and the dissemination and use of the guidelines will need to be evaluated as the authors point out. The main barriers will be time and resources, expertise development and follow up of results.

So why are these guidelines particularly valuable now? Looking back over the past 15 years, the increasing life expectancy of patients has led to new challenges. Improvements in diagnosis and care mean that CF clinics have greater numbers of patients. At diagnosis, the information patients/parents receive is more complex than 15 years ago. Gene-specific therapy means that we must discuss with families the importance of genotype for future therapy at an early stage. In addition, even though life expectancy has improved and hope (about new medicines) has increased, patients/parents are still informed about untimely death, comorbidities, transplantation, etc. Treatment modalities have become more diverse and patients/parents are facing more complex individual treatment plans compared with families 15 years ago. New medicines (eg, dornase alfa and inhaled antibiotics) have improved life expectancy and quality, but they have also increased the burden of treatment.

Overall, the emotional wellbeing of an increasing number of patients/parents is affected by many more factors today compared with issues that families had to deal with in the past. This complexity makes patients/parents more vulnerable and prone to psychological problems. The diversity of psychological support required is far greater than 15 years ago and the number of patients needing this support has expanded extensively.

Here the new mental health guidelines offer us a solution by being a valuable 


\section{Editorial}

addition to the range in psychosocial care services for patients and families. Guidelines can help a team to feel assured that the psychosocial wellbeing of all patients will be evaluated, at least on a basic level once a year. In addition, the guidelines will not and cannot jeopardise the important continuous and individual contacts psychosocial health care providers have with patients. On the contrary, they should help to identify patients/ parents in need of this help and allow timely intervention.

In future, the guidelines need to be assessed for usefulness in the day-to-day clinic. Time and resources are scarce and for some clinics only some recommendations can be implemented to start with. The guidelines are to be used to improve individual (family) care for patients and parents. In time, the data can also be used (through registries, benchmarking and quality improvement initiatives) to further understand the long-term interactions between disease course and mental wellbeing. For example, does providing psychosocial support based on screening improve mental health in patients with CF and does this interact with illness progression? The TIDES study has shown considerable anxiety and depression in patients with CF; let us hope we can turn the tide.

Competing interests None declared.

Provenance and peer review Commissioned; internally peer reviewed.

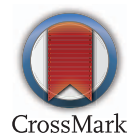

To cite Havermans T, Staab D. Thorax 2016;11:1-2.

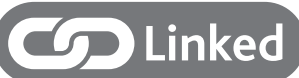

http://dx.doi.org/10.1136/thoraxjnl-2015-207488

Thorax 2016;71:1-2.

doi:10.1136/thoraxjnl-2015-208076

\section{REFERENCES}

1 Quittner AL, Abbott J, Georgiopoulos AM, et al. International Committee on Mental Health in Cystic Fibrosis: Cystic Fibrosis Foundation and European Cystic Fibrosis Society consensus statements for screening and treating depression and anxiety. Thorax 2016;71:26-34.

2 Standards CFTC, Accreditation G. Standards for the clinical care of children and adults with cystic fibrosis in the UK 2001. London: UK Cystic Fibrosis Trust, 2001.

3 Kerem E, Conway S, Elborn S, et al. Standards of care for patients with cystic fibrosis: a European consensus. J Cyst Fibros 2005:4:7-26.

4 Smyth AR, Bell SC, Bojcin S, et al. European Cystic Fibrosis Society Standards of Care: best practice guidelines. J Cyst Fibros 2014;13(Suppl 1):S23-42.

5 Abbott J, Elborn JS, Georgiopoulos AM, et al. Cystic Fibrosis Foundation and European Cystic Fibrosis Society Survey of cystic fibrosis mental health care delivery. J Cyst Fibros 2015;14:533-9.

6 Quittner AL, Goldbeck L, Abbott J, et al. Prevalence of depression and anxiety in patients with cystic fibrosis and parent caregivers: results of the International Depression Epidemiological Study across nine countries. Thorax 2014;69:1090-7.

7 Robinson KA, Saldanha IJ, McKoy NA. Development of a framework to identify research gaps from systematic reviews. J Clin Epidemiol 2011;64:1325-30. 